\title{
Plant Recombinant Human Collagen Type I Hydrogels for Corneal Regeneration
}

\author{
Michel Haagdorens ${ }^{1,2}$ (D) $\cdot$ Elle Edin $^{3,4,5}$ (D) $\cdot$ Per Fagerholm $^{6}$ (D) $\cdot$ Marc Groleau $^{4,5}$ (D) Zvi Shtein $^{7,8} \cdot$ Artūras Ulčinas $^{9}$ (D) \\ Amit Yaari ${ }^{7,8} \cdot$ Ayan Samanta $^{10} \cdot$ Vytautas Cepla $^{9,12}$ (1) - Aneta Liszka ${ }^{6} \cdot$ Marie-José Tassignon $^{1,2}$ (1) • \\ Fiona Simpson $^{3,4,5}$ (D) - Oded Shoseyov ${ }^{7,8,13}$ (D) Ramūnas Valiokas $^{9}$ (D) - Isabel Pintelon ${ }^{11}$ (D) \\ Monika Kozak Ljunggren $^{6}$ (D) - May Griffith ${ }^{3,4,5}$ (D)
}

Published online: 6 August 2021

(C) The Author(s) 2021
Abstract
Purpose To determine feasibility of plant-derived recombinant human collagen type I (RHCI) for use in corneal regenerative implants Methods RHCI was crosslinked with 1-ethyl-3-(3-dimethyl aminopropyl) carbodiimide (EDC) and N-hydroxysuccinimide (NHS) to form hydrogels. Application of shear force to liquid crystalline RHCI aligned the collagen fibrils. Both aligned and random hydrogels were evaluated for mechanical and optical properties, as well as in vitro biocompatibility. Further evaluation was performed in vivo by subcutaneous implantation in rats and corneal implantation in Göttingen minipigs.
Results Spontaneous crosslinking of randomly aligned RHCI (rRHCI) formed robust, transparent hydrogels that were sufficient for implantation. Aligning the RHCI (aRHCI) resulted in thicker collagen fibrils forming an opaque hydrogel with insufficient transverse mechanical strength for surgical manipulation. rRHCI showed minimal inflammation when implanted subcutaneously in rats. The corneal implants in minipigs showed that rRHCI hydrogels promoted regeneration of corneal epithelium, stroma, and nerves; some myofibroblasts were seen in the regenerated neo-corneas.
Conclusion Plant-derived RHCI was used to fabricate a hydrogel that is transparent, mechanically stable, and biocompatible when grafted as corneal implants in minipigs. Plant-derived collagen is determined to be a safe alternative to allografts, animal collagens, or yeast-derived recombinant human collagen for tissue engineering applications. The main advantage is that unlike donor corneas or yeast-produced collagen, the RHCI supply is potentially unlimited due to the high yields of this production method.
Lay Summary A severe shortage of human-donor corneas for transplantation has led scientists to develop synthetic alternatives. Here, recombinant human collagen type I made of tobacco plants through genetic engineering was tested for use in making corneal implants. We made strong, transparent hydrogels that were tested by implanting subcutaneously in rats and in the corneas
Michel Haagdorens and Elle Edin contributed equally to this work.
Monika Kozak Ljunggren
Monika.Ljunggren@liu.se
$\triangle$ May Griffith
May.Griffith@umontreal.ca
Isabel Pintelon
isabel.pintelon@uantwerpen.be
1 Department of Ophthalmology, Visual Optics and Visual Rehabilitation, University of Antwerp, Antwerp, Belgium
2 Department of Ophthalmology, Antwerp University Hospital, Antwerp, Belgium
3 Department of Ophthalmology and Institute of Biomedical Engineering, University of Montreal, Montreal, Quebec, Canada
4 Maisonneuve-Rosemont Hospital Research Centre, Montreal, Quebec, Canada
5 CHUM Research Centre, Montreal, Quebec, Canada
6 Department of Clinical and Experimental Medicine, Linköping University, Linköping, Sweden
7 The Robert H. Smith Faculty of Agriculture, Food and Environment, Rehovot, Israel
8 The Center for Nanoscience and Nanotechnology, The Hebrew University of Jerusalem, Jerusalem, Israel
9 Department of Nanoengineering, Center for Physical Sciences and Technology, Vilnius, Lithuania
10 Department of Chemistry-Ångström Laboratory, Uppsala, Sweden
11 Laboratory of Cell Biology and Histology, Antwerp University, Antwerp, Belgium
12 Ferentis UAB, Vilnius, Lithuania
13 CollPlant Ltd., Ness-Ziona, Israel 
of minipigs. We showed that the plant collagen was biocompatible and was able to stably regenerate the corneas of minipigs comparable to yeast-produced recombinant collagen that we previously tested in clinical trials. The advantage of the plant collagen is that the supply is potentially limitless.

Keywords Plant collagen · Cornea regeneration · Limbal stem cells · Tissue engineering $\cdot$ Recombinant human collagen type 1

\section{Introduction}

The human cornea is the transparent front of the eye that focuses incoming light onto the retina for vision. Its cellular layers comprise an outermost epithelium, a middle stroma composed of collagenous extracellular matrix (ECM) with interconnected keratocytes, and an innermost endothelium. It is heavily innervated, but avascular. Damage or disease leading to permanent transparency loss or surface distortion can result in corneal blindness. Historically, the only widely used treatment for restoring vision is human donor cornea (HDC) transplantation. However, a serious donor shortage has left an estimated 12.7 million patients worldwide waiting for corneal transplants [1]. Moreover, the outcome of conventional corneal transplantation is limited by immune rejection, ocular infection, and premature graft failure. Artificial corneas in the form of prostheses have been introduced in clinics, most notably the Boston KPro, AlphaCor, and osteo-odonto-keratoprosthesis (OOKP) [2-4]. All three prostheses are associated with potentially serious side effects and are regarded as a last resort treatment for patients with severe pathology or previous conventional graft failures [5]. Given the shortcomings of conventional corneal transplantation and insufficient performance of prostheses, various research groups have focused on the development of a range of corneal replacements [6,7].

In 2010, Fagerholm et al. published the 2-year results of a clinical trial describing the first-in-human in situ tissue regeneration of the cornea. Tissue regeneration was achieved by implanting cell-free, bio-responsive recombinant human collagen type III (RHCIII) hydrogels [8]. RHCIII was selected as a homogenous, non-xenogeneic protein that minimizes the risk of allergic reactions to animal source collagens, or zoonotic disease transmission [9]. The results of the RHCIII hydrogels are promising, but the human cornea is primarily composed of type I collagen. Here, we examine the biocompatibility and efficacy of recombinant human collagen type I (RHCI) collagen hydrogels as implants in minipig corneas, compared to syngeneic grafts.

The RHCI implants designed for this study are the first to use plant-derived type I collagen. A prior comparison of RHCI and RHCIII has been made, but both collagens were produced in Pichia pastoris. Protein production in yeast is a fermentation-based process that requires the additional expression of two enzymes: prolyl 4-hydroxylase (P4H) and pepsin. PH4 is the enzyme that catalyzes the formation of 4-hydroxyproline amino acids from proline [10]. The 4-hydroxyproline amino acids are needed for stable triple helix formation. The recombinant pepsin is needed to cleave the telopeptides from the full-length protein before use [9]. This process is inherently limited by the fermentation batch size.

Plant-derived RHCI is generated from genetically modified tobacco plants, which allows the production of theoretically unlimited quantities of protein and supports agricultural development. The two genes encoding RHCI were co-expressed in tobacco plants with $\mathrm{P} 4 \mathrm{H}$ and lysyl hydroxylase 3 (LH3) enzymes that were responsible for key post-translational modifications of the RHC [11]. Tobacco plants co-expressing all five vacuole-targeted proteins generated intact RHCI pro-collagen with stable triple helical structures. Tobacco plant-derived RHCI was shown to have equivalent biofunctionality to human tissue-derived collagen in other applications [11-13]. We recently reported that RHCI hydrogels were fully biocompatible with corneal cells and served as excellent substrates for corneal limbal epithelial stem cells, showing comparable efficacy to human amniotic membrane, the current gold standard for their culture [14].

Previous studies of collagen implants did not include attempts to align the collagen fibrils, although there have been numerous reports of attempts to align collagen fibrils for use as corneal implants, citing the need for transparency and mechanical strength $[15,16]$. Here, we examined the properties of RHCI with aligned and random fibrils in vitro and their biocompatibility in vivo in a rat model. The RHCI hydrogel showing the most optimal properties for use as corneal implants was tested in a minipig model, where its efficacy was compared to that of allografting, simulating donor cornea transplantation.

\section{Materials and Methods}

The study followed the tenets of the Declaration of Helsinki and was approved by the Antwerp University Hospital Ethical Committee (EC: 14/30/319) for use of human amniotic membrane. Animal experiments in rats and Gottingen minipigs were performed in accordance with the Association for Research in Vision and Ophthalmology Statement for the Use of Animals in Ophthalmic and Vision Research, and the Swedish Animal Welfare Ordinance and the Animal Welfare Act, after ethical approval was acquired from the local Linkoping ethical committee (Linköpings Djurförsöksetiska Nämnd). 


\section{Materials}

All inorganic salts, enzymes, basic chemicals, and reagents were purchased from Sigma-Aldrich (St. Louis, MO, USA), unless stated otherwise. Plant-derived RHCI was provided by Collplant (Ness Ziona, Israel).

\section{Recombinant Human Collagen Hydrogels}

\section{RHCl Hydrogels}

To fabricate RHCI hydrogels with random fibrillogenesis (rRHCI), we use the protocol described in Haagdorens et al. [14]. Briefly, a $30 \% \mathrm{v} / \mathrm{v}$ solution of tobacco-derived RHCI in $10 \mathrm{mM} \mathrm{HCl}$ was prepared by diluting with $100 \%$ ethanol. Fibrillogenesis was initiated by addition of $160 \mathrm{mM}$ sodium phosphate buffer that was adjusted to $\mathrm{pH} 7.5$ with $100 \mathrm{mM}$ $\mathrm{NaOH}$. The buffer was added at a ratio of $1: 10 \mathrm{v} / \mathrm{v}$ to the original collagen- $\mathrm{HCl}$ volume. The solution was stirred for $2 \mathrm{~h}$ at $25^{\circ} \mathrm{C}$; after which, water-diluted EDC and NHS were added to attain a $50 \mathrm{mM}$ 1-ethyl-3-(3-dimethyl aminopropyl) carbodiimide (EDC) and $100 \mathrm{mM}$ N-hydroxysuccinimide (NHS) concentration. The collagen solution was then stirred for $24 \mathrm{~h}$ at $4^{\circ} \mathrm{C}$ on a magnetic stirrer at $200 \mathrm{rpm}$. The solution was then transferred into a $50-\mathrm{mL}$ tube for 6 cycles of washing to remove all excess EDC/NHS. Each cycle consisted of centrifuging the mixture at full-speed (5000 rpm, $10 \mathrm{~min})$, discarding the supernatant and resuspending the collagen in $40 \mathrm{~mL}$ distilled water (DW). During the final wash cycle, the RHCI suspension was dispensed into a rectangular Teflon mold $(4.5 \times 4.5 \mathrm{~cm})$ for in vitro characterization or into curved corneal-shaped and corneal-sized molds $(10 \mathrm{~mm}$ diameter, $500-\mu \mathrm{m}$ thick) as implants. The collagen was then air-dried under a sterile hood and immersed in 100\% ethanol to promote detachment from the mold. The gels were stored in $100 \%$ ethanol until further use. Gels were rehydrated by five 2-h soaks in phosphate-buffered saline (PBS).

\section{RHCl Hydrogels with Aligned Collagen Fibrils}

For production of aligned RHCI membranes (aRHCI), shear force was applied to the collagen solution prior to fibrillogenesis and chemical crosslinking. Shear force was effected using a motorized automatic film applicator, Elcometer 4340 (Elcometer Ltd, Manchester, UK) and an Elcometer 3570 "Doctor Blade" that served as the orthogonal force. After ethanol dilution, the acidic RHCI was cast onto a glass plate that was mounted in the Elcometer. The glass plate had previously been surface-coated with a hydrophobic siloxane solution. After collagen casting, shear force was applied at a constant speed of $0.02 \mathrm{~m} / \mathrm{s}$ and a thickness of $50 \mu \mathrm{m}$. After the RHCI had spread, the glass plate was immediately lifted from the Elcometer and immersed in a bath of fibrillogenesis buffer. This was performed very carefully in order not to disrupt the collagen membrane. Membranes were then crosslinked for $24 \mathrm{~h}$ in fibrillogenesis buffer and $50 \mathrm{mM}$ EDC and $100 \mathrm{mM}$ NHS. Crosslinked membranes were then rinsed four times in DW and peeled from the glass plate. Hydrogels were kept in $100 \%$ ethanol until further use.

\section{Human Amniotic Membrane}

Human amniotic membrane (HAM), prepared following our previous protocol, was used as a benchmark [14]. Written informed consent was collected from the donors, women undergoing scheduled cesarean section. Briefly, HAM was washed in an antibiotic cocktail and then flattened onto sterilized nitrocellulose filter paper, epithelial side up. The paper-supported HAM was then cut into $5 \mathrm{~cm} \times 5 \mathrm{~cm}$ pieces and cryopreserved at $-80^{\circ} \mathrm{C}$ in $50 \%$ Dulbecco's Modified Eagle Medium (DMEM), 50\% glycerol. The membrane was quarantined for 4 weeks during which time sterility and serology testing were performed to confirm safety. Prior to use, HAM was thawed, epithelial cells removed, and the cell-free membrane was washed. For use, HAM is oriented spongy layer up, after which excessive water is mechanically expelled using cell scrapers. For stabilization, HAM was mounted "spongy layer" down in a CellCrown or interlockable ring [17].

\section{Physical and Mechanical Characterization}

To measure water content, samples were DW equilibrated for $24 \mathrm{~h}$. Water content of samples was determined by weighing membranes that were blotted dry to remove excess surface liquid to obtain the wet weight $\left(W_{0}\right)$. These samples were then dried in a drying oven $\left(60^{\circ} \mathrm{C}\right)$ for $24 \mathrm{~h}$ to obtain the dry weight $(W)$. Equilibrated water content of hydrogels $\left(W_{\mathrm{t}} \%\right)$ was obtained according to the following equation: $W_{\mathrm{t}} \%=\left(W_{0}-W\right) /$ $W_{0} \times 100 \%$.

The refractive index (RI) of samples was measured of fully hydrated membranes using an Abbe refractometer (Model C10, VEE GEE Scientific Inc., Kirkland, WA, USA) at $21^{\circ}$ $\mathrm{C}$ with DW as the calibration agent.

To test membrane light transmission, 6-mm acellular discs of the sample were punched out and placed in a glass bottom 96-well plate. Using the previously published protocol [18], light absorbance was measured at room temperature for specific wavelengths of the visual spectrum $(405,450,490,530$, and $630 \mathrm{~nm}$ ) with the VICTOR $^{3}$ microplate reader (PerkinElmer). Percentage of light transmittance was calculated with the following equation: transmission $(\%)=10^{(2}$ - absorbance). Wells mounted with $100 \mu \mathrm{L}$ of trypan blue or DW served as negative and positive controls, respectively. Transmittance values were normalized to demineralized water. 
Oscillatory rheology was performed on a Discovery Hybrid Rheometer (DHR2; TA Instruments, Sollentuna, Sweden) using 8-mm diameter, parallel-plate, stainless steel geometry. Frequency sweeps were performed at a constant shear strain amplitude of $0.267 \%$ at $25^{\circ} \mathrm{C}$.

Tensile testing of the membrane samples was performed on a vertical stress-strain testing device (Instron 3345, Canton, MA, USA) fitted with a $100 \mathrm{~N}$ load cell. Testing was performed at a $2 \mathrm{~mm} / \mathrm{min}$ rate. Membrane samples where cut into $4 \times 0.5$-cm-long strips and fixed at both ends to a 1 mm-thick polypropylene frame. The ultimate tensile strength (UTS) was calculated based on the area of the sample (sample thickness measured with optical coherence tomography; RTVue 100-2, Optovue, California, USA).

\section{Hydrogel Stability and Susceptibility to Enzymes and Microbial Attack}

The stability of the hydrogels was tested against in vitro degradation by collagenase enzyme [19]. In brief, samples $(n=3)$ equilibrated in Tris- $\mathrm{HCl}$ buffer $(0.1 \mathrm{M}, \mathrm{pH} 7.4)$ were incubated in $5 \mathrm{~mL} 5 \mathrm{U} / \mathrm{mL}$ type I collagenase dissolved in Tris- $\mathrm{HCl}$ at $37^{\circ} \mathrm{C}$. The undigested mass was weighed at time $0\left(W_{0}^{\prime}\right)$ and every $60 \min \left(W_{t}^{\prime}\right)$ until the sample was completely digested. At every interval, surface water was blotted away, and samples were weighed three times independently using an ultra-microbalance (SE2, Sartorius, Göttingen, Germany). The percentage of mass remaining after digestion was calculated according to the following equation: Residual mass $(\%)=\left(W^{\prime} / W^{\prime}{ }_{0}\right) \times 100 \%$. As a control, HAM was incubated in Tris- $\mathrm{HCl}$ buffer only and weighed at the same time points.

For determination of attack by common bacteria causing blindness, such as Gram-negative Pseudomonas aeruginosa, fully hydrated samples equilibrated in PBS were cut into $1 \mathrm{~cm}^{2}$ pieces. Hydrogels were mounted in a 24 well-plate and fixed using a CellCrown. As previously described by Dravida et al. [20], a dilution of $10^{-1}$ of two P. aeruginosa strains (ATCC 15442 and ATCC 9027) was made in Tryptic Soy Broth (TSB). Using a 22 gauge needle, $100 \mu \mathrm{L}$ solution was injected into the scaffold ( $n=3$ for each ATCC strain). The inoculated scaffolds were incubated in an oven $\left(37^{\circ} \mathrm{C}\right)$ for $24 \mathrm{~h}$. The samples were homogenized in PBS using a tissue grinder. The homogenized extracts were diluted in the range of $10-10^{-8}$ and plated on agar (TSA). The number of colony-forming units (CFU)/mL was determined $24 \mathrm{~h}$ later. To have sufficiently thick amnion to inject the solution, mechanical scraping of the spongy layer was not performed for this experiment. Injection of $100 \mu \mathrm{L}$ bacterial solution in $10 \mathrm{~mL}$ TSB and $10 \mathrm{~mL}$ PBS served as negative and positive controls respectively.

\section{Ultrastructural Characterization}

\section{Atomic Force Microscopy}

Membrane samples of $10 \times 10 \mathrm{~mm}^{2}$ were cut out and placed onto a rigid support carrier. The carrier, a glass slide that had functional aldehyde surface groups (developed in-house), was then immersed in PBS to allow full rehydration of the sample prior to atomic force microscopy (AFM) imaging. HAM samples received additional gentle mechanical scraping with cell scrapers to remove excess spongy layer, thus exposing the underlying membrane and reducing height variation. While imaging, samples were kept hydrated. AFM imaging was carried out in PBS by a NanoWizard 3 (JPK Instruments, Germany) system using NSC35 probes (MicroMasch, Germany) and the force curve acquisition-based Quantitative Imaging ${ }^{\mathrm{TM}}(\mathrm{QI})$ mode. Topography images were generated with the setpoint height channel in the QI-mode, setpoint force 5-10 of nN. Fibril diameters were estimated from height cross-sections extracted from the AFM images. Full-width at half-maximum was measured where the image resolution was sufficient. In other cases (e.g., rRHCI characterized by very fine fibrils), a trained operator's estimates were used.

\section{Electron Microscopy}

Samples of biomaterials were fixed and prepared for scanning electron microscopy (SEM) and transmission electron microscopy (TEM) as we previously described in Haagdorens et al. [14]. Gold sputter-coated samples were imaged on a SEM 515 Microscope (Philips, Eindhoven, The Netherlands) for surface features [14]. Ultra-thin sections were cut from osmicated samples embedded in EMbed 812 (Electron Microscopy Sciences, Hatfield, PA). Ultrathin sections were stained with lead citrate and examined using a Tecnai G2 Spirit Bio Twin Microscope (FEI, Eindhoven, The Netherlands) at $120 \mathrm{kV}$.

\section{In vivo Biocompatibility Testing in Rats}

Random RHCI hydrogels, $11 \mathrm{~mm}$ in diameter and 1-mm thick, were implanted subcutaneously in rats (following ISO standard 10993-6:2007). Denuded HAM was also implanted as a comparison for RHCI. To achieve samples of sufficient thickness, 7 layers of HAM were stacked upon each other and sealed with fibrin glue (Tisseel, Baxter, Deerfield, IL, USA). Clotted fibrin sealant molded into $1-\mathrm{cm}^{3}$ blocks were implanted as a control. In total, 5 collagen hydrogels, 4 HAM samples, and 3 fibrin clots were implanted subcutaneously. Two sham surgeries were performed to ensure that the skin healing process does not affect the outcome of biocompatibility testing. Postoperatively, extended assessment of health conditions of animals was performed based on an observational grading scheme (not shown). 
At 3 months post-implantation, the animals were euthanized, and the implants and surrounding tissues were collected. Each rRHCI samples was subdivided into quarters for analysis. Quarters one and two were fixed in formalin, then embedded in paraffin or O.C.T. compound, respectively for histology and immunohistochemistry. One quarter was fixed in glutaraldehyde for EM. The final quarter was not analyzed. Recovered HAM, fibrin, and sham samples were similarly processed for histology and immunohistochemistry.

Non-consecutive paraffin sections, cut at 7- $\mu \mathrm{m}$ thick, were stained with hematoxylin and eosin (H\&E) for histological evaluation. Based on H\&E staining, two slides, each containing three tissue sections of the same sample, were used to assess biocompatibility as per Table S1 (adapted from ISO10993-6:2007 Table E3). According to this grading table, the test sample was considered a non-irritant (0 up to 2.9), slight irritant (3.0 up to 8.9), moderate irritant (9.0 up to 15.0 ), or severe irritant ( $>15)$. Grading was performed double-blinded. In brief, each H\&E section was graded at five different sites, two at each respective end, and one in the middle of the sample. If remnants of the implant could be identified, the area of grading was at the junction of the sample and surrounding tissue.

Cryosections, 7- $\mu \mathrm{m}$ thick, were stained with antibodies to identify plasma cells (anti-syndecan-1), granulocytes (anti-myeloperoxidase), macrophages (anti-CD68), T-lymphocytes (anti-CD3), blood vessels (anti-vWF), and activated myofibroblasts (anti- $\alpha \mathrm{SMA}$ ) as previously described (Table S2) [38]. Immunofluorescent (IF) images were recorded using an inverted LSM-800 Zeiss confocal microscope (LSM800, Carl Zeiss Microscopy, Germany), and the percentage of positive staining area per view was calculated for each sample using the ImageJ software (National Institutes of Health, Bethesda, MD, USA). In each stained sample, the analysis was performed for three IF images at standard locations (left end, middle, and right end of the sample). A Kruskal-Wallis nonparametric test was used with a Dunn's multiple comparisons post hoc test. Statistical analysis was performed in GraphPad Prism 8.4.3. $P \leq 0.05$ was considered significant.

\section{In vivo Efficacy Testing as Corneal Implants in Minipigs}

To test in vivo safety and efficacy of corneal regeneration, the implants were evaluated in Göttingen minipigs, which are genetically uniform. Four animals were used in each treatment group. The left eyes of the pigs underwent surgical treatment, while the right eyes served as unoperated controls. All animals were subjected to anterior keratoplasty. The central section of corneal epithelium and stroma was removed to a depth of $500 \mu \mathrm{m}$ using a 6.5-mm surgical trephine and a scalpel. Solid rRHCI gels were grafted into the wound bed, covered with HAM, and secured in place using interrupted sutures. In the control group, cornea excised from the other pigs in this study was used as implants, being secured with interrupted sutures. These were syngeneic grafts rather than conventional allografts, as they are from genetically identical donors and recipients. The syngeneic grafts were secured using interrupted sutures. All operated eyes received corticosteroids and antibiotics in the form of topical dexamethasone/tobramycin eye drops (Tobrasone, Alcon, Sweden). The dexamethasone/ tobramycin was applied 3 times daily for the first 5 postoperative days. The pigs were monitored daily for any eye-related complications for the entire study duration. Clinical exams were performed pre-operation, and then at 6 weeks, and 3, 6, 9 , and 12 months post-operation according to protocols as we described in McTiernan et al. [21]. Briefly, all operated and control corneas were examined by slit lamp biomicroscopy (for haze, redness), anterior segment optical coherence tomography (for corneal changes including thickness), Schirmer's tear test (for tear production), and aesthesiometry (for touch sensitivity). At the endpoint of 12 months post-operation, all animals were euthanized according to animal facility guidelines, and tissue samples of both operated and contralateral eyes were collected.

Samples from implanted and control corneas were processed for TEM as described in McTiernan et al. [14, 21, 22]. In brief, samples were prepared for TEM by fixing a quarter of each cornea in $2.5 \%$ glutaraldehyde solution in $0.1 \mathrm{M}$ sodium cacodylate buffer ( $\mathrm{pH} 7.4$ ). After fixing, the quarters were cut into 1-mm wide strips. The strips were postfixed in $1 \% \mathrm{OsO} 4$ solution for $2 \mathrm{~h}$, dehydrated through an ethanol series and embedded in EMbed 812 (Electron Microscopy Sciences, Hatfield, Pennsylvania). Ultrathin sections stained with lead citrate were imaged on a Tecnai G2 Spirit Bio Twin Microscope (FEI, Eindhoven, The Netherlands) at $120 \mathrm{kV}$.

In vivo confocal microscopy examinations were performed before surgery and at 3, 6, 9, and 12 months post-surgery. Images taken were used for nerve counts as described in McTiernan et al. [21]. When nerves where observed, they were defined as bright, slender, straight, or branched structures and were sufficiently different from the background intensity level. NeuronJ, a nerve tracing and analysis software, and FIJI were used together for measuring the total length of the observed nerves [23]. Corneal nerve densities were based on averaging the highest nerve density for each treatment group and time point from individual images.

\section{Statistics}

For all tests of hydrogels, a minimum of three samples was used in evaluations. For the in vivo subcutaneous studies, 5 RHCI hydrogels, 4 HAM samples, and 3 fibrin clots were implanted. The additional RHCI and HAM samples were implanted just in case animals died from unrelated causes, as these samples were more difficult to obtain. As all animals 
remained healthy and survived, so, all samples were analyzed for potential adverse immune cell infiltration or activation by differential cell counts followed by a Kruskal-Wallis nonparametric test as described above.

The unit of analysis for the in vivo study in minipigs was the eye (RHCI $n=4$, syngeneic graft $n=4$, unoperated $n=8$ ). Clinical variables over time (Fig. 3a-f) were analyzed with a mixed effects model with matched stacking, no assumption of sphericity, and $\alpha=0.05$ using a post hoc Tukey's multiple comparison test. Statistical analysis and graphing were performed using GraphPad Prism v8.4.3 (GraphPad Software LLC, San Diego, CA, USA).

\section{Results}

\section{Physical and Mechanical Properties of RHCI Hydrogels}

The properties of the aligned and random RHCI hydrogels, compared to HAM, are summarized in Fig. 1. Both types of collagen hydrogel and control HAM displayed a RI that approximates that of the human cornea (1.37-1.38). The rRHCI hydrogels allowed for light transmittance within the visual spectrum of over $91 \%$ (Fig. S1) while aRHCI hydrogels showed only $37 \%$ light transmission, appearing opaque (Fig. $\mathrm{S} 1,1 \mathrm{a})$. Figure 1a also shows that the aRHCI hydrogels were prone to splitting along the direction of the collagen fibrils. HAM, which served as a control, showed light transmission of $66 \%$. The opacity of the aRHCI hydrogels precluded its use for corneal implants, while it was tested for toxicity and biocompatibility as subcutaneous implants in rats, it was not tested as corneal implants in vivo. The rRHCI hydrogels used for corneal implantation, however, was robust, pliable, and easy to manipulate when molded into corneal-shaped and corneal-sized implants (Fig. S2).

Both aligned and random hydrogels, as well as HAM, showed a predominantly elastic, rather than viscous behavior with storage moduli much higher than loss moduli. However, due to the very low thickness of HAM, reliable data from oscillatory rheology was not possible to obtain and therefore, eliminated from further comparisons. The $\mathrm{G}^{\prime}$ of rRHCI gels was found to be $2.9 \pm 0.03 \mathrm{kPa}$, whereas that of aRHCI hydrogels was found to be $0.383 \pm 0.03 \mathrm{kPa}$ indicating a 10 -fold higher stiffness for the rRHCI (Fig. S3). The aRHCI hydrogels have slightly higher water content compared to their random counterparts. Moreover, the $\mathrm{G}^{\prime \prime}$ of the rRHCI hydrogels was found to be $0.158 \pm 0.036 \mathrm{kPa}$ resulting in a $\tan \delta$ of $0.05 \pm 0.01$. Stress strain analysis shows that both RHCI hydrogels display stress and strain values that are inferior to that of HAM membrane. These results reveal that HAM displayed roughly three times greater energy absorption compared to
Fig. 1 Structural properties of RHCI hydrogels compared to human amniotic membrane (HAM). a Aligned hydrogels are semi-translucent and have the tendency of splitting unidirectionally. b Random collagen hydrogels are optically transparent. c Control HAM serves as reference. $\operatorname{AFM}(\mathbf{d}-\mathbf{f})$ and SEM (g-i) of hydrogels, showing that in random hydrogels, very fine collagen fibrils were present, resulting in a smooth surface as revealed by SEM. Cross-sectional TEM of the hydrogels with aligned fibers (j) showed thick RHCI fibers (arrow) interspersed with thin collagen fibrils (arrowhead). The collagen fibers show unidirectional alignment, whereas the interpenetrating fibrils are randomly dispersed (k). Fibers were of varying thickness, 75-200-nm diameter. In random hydrogels, only fine collagen fibrils are seen, although some clumping was seen in the AFM and TEM images. I Collagen fibers were randomly oriented in HAM, with fibers showing distinct collagen banding (inset). The inset table shows the optical and mechanical characteristics of RHCI hydrogels and human amniotic membrane (HAM). *The stress test was not performed on the aligned RHCI hydrogels. **not performed

random RHCI hydrogels. Young's modulus indicates that the latter are much stiffer than the HAM. The aRHCI hydrogels were inherently too weak to allow stress-strain analysis

Collagenase degradation assays showed that aligned and random RHCI hydrogel degradation was complete by $8 \mathrm{~h}$ and $16 \mathrm{~h}$ respectively (Fig. S4). The degradation of HAM was slower, with complete degradation occurring after $24 \mathrm{~h}$. Inoculation with $P$. aeruginosa showed that RHCI hydrogels, irrespective of fibril alignment or not, were 50- to 100 -fold more resistant to microbial contamination for both strains of Pseudomonas (Table S3) compared to HAM. We recently showed in Haagdorens et al. that RHCI hydrogels were biocompatible with human corneal epithelial cells and supported their growth [14].

\section{Ultrastructure}

Surface scanning by AFM (Fig. 1d-f) confirmed the random arrangement of fibrils in rRHCI hydrogels, and the unidirectional alignment of the RHCI fibrils in the shear-mediated preparation of aRHCI. It was noted that the collagen fibrils in the aRHCI hydrogels are thicker $(93.8 \pm 30.6 \mathrm{~nm}$ in diameter) than the fibrils in the rRHCI hydrogels $(23.6 \pm 10.4 \mathrm{~nm}$ in diameter $)$. This showed that the fibrils had assembled into distinct thicker bundles of fibrils or collagen fibers. Fibrils in the random hydrogel were very fine, but also matted in areas. HAM fibrils were more similar to aRHCI in thickness $(112.6 \pm 61.7 \mathrm{~nm}$ in diameter $)$.

SEM (Fig. 1g-i) confirmed the unidirectional collagen orientation in the surface of aHCI hydrogels. The rRHCI hydrogels showed a smooth surface. SEM imaging of HAM revealed complete removal of amniotic epithelium and exposure of the underlying basement membrane. At the amniotic basement membrane, remaining cellular debris and extracellular matrix $(\mathrm{ECM})$ could be seen between collagen fibers. 
$\mathrm{aRHCl}$
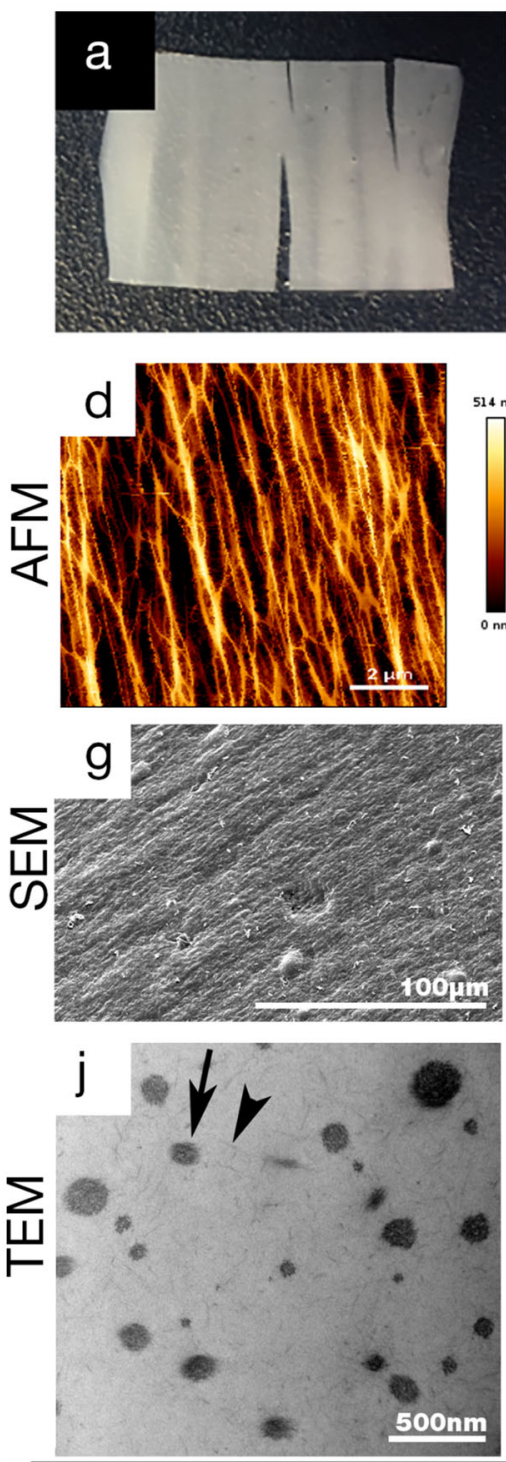

\begin{tabular}{lllll}
\hline Properties & aRHCI & rRHCI & HAM & Human Cornea $^{1}$ \\
\hline Water content (\%) & $92.21 \pm 0.007$ & $89.21 \pm 0.003$ & $87.68 \pm 0.018$ & 78 \\
$\begin{array}{l}\text { Transmission (\%) } \\
\text { Tensile strength (MPa) }\end{array}$ & 37.1 & 91.5 & 65.9 & 87 \\
Elongation at break (\%) & $2.67 \pm 0.8$ & $7.6 \pm 2.4$ & $3.81 \pm 0.4$ \\
$\begin{array}{l}\text { Young's Modulus } \\
(\mathrm{MPa})\end{array}$ & $*$ & $28 \pm 6$ & $68 \pm 10$ & $* *$ \\
\end{tabular}

Amniotic membrane
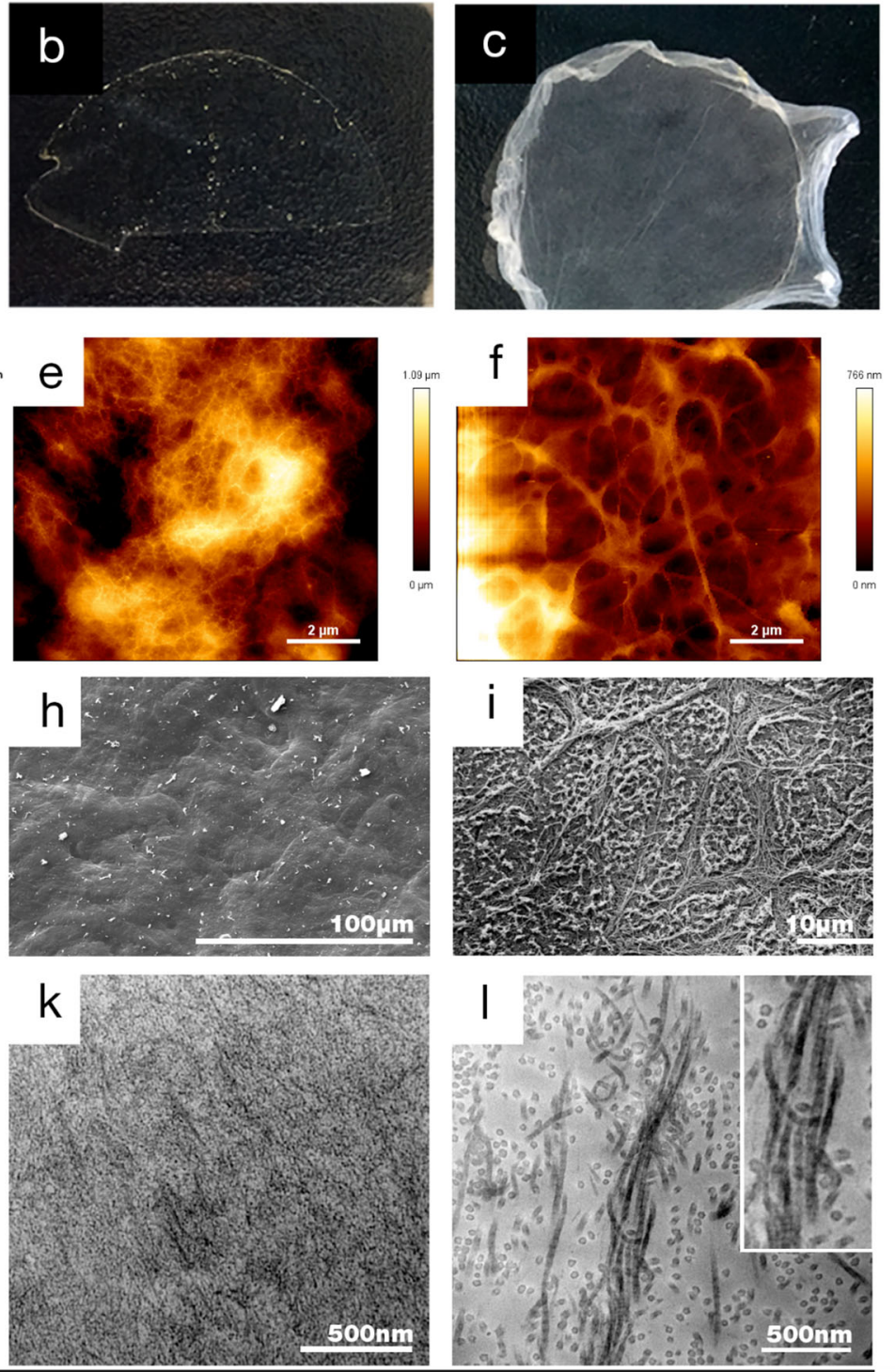

1: Fagerholm P, Lagali NS, Ong JA, Merrett K, Jackson WB, Polarek JW et al. Biomaterials. 2014;35(8):2420-7.

TEM of rRHCI hydrogels confirmed the presence of fine collagen fibrils distributed randomly throughout the sample (Fig. 1k) as revealed by AFM. In the aRHCI, the fibrils formed thick collagen bundles (Fig 1j) as seen in the cross-sectional views. They showed unidirectional alignment in contrast to the interpenetrating thin collagen fibrils. 


\section{In vivo Biocompatibility in Rats}

After subcutaneous implantation of the rRHCI hydrogels in rats, the animals did not exhibit any general or implantation-site adverse symptoms. Upon recovery of the implants, there were no clear macroscopic signs of implant degeneration, and the RHCI hydrogels could easily be distinguished from the surrounding tissues. Based on grading of the H\&E-stained samples (Fig. 5a-f), RHCI hydrogels were ruled as being slightly irritant, with an average grading score of 7.33 , well comparable to controls with scores of 7.63 for fibrin, 9.75 for HAM and 7.93 for sham (Table S4). Transmission electron microscopy images of implanted hydrogels showed isotropic collagen fibers interspersed with capillaries and small blood vessels, along with fibroblasts (Fig. 2b-e). Microscopically, some degree of disintegration was evident, and the RHCI hydrogels were mainly infiltrated by lymphocytes, fibroblasts, and macrophages with some ingrowth of capillary vessels (Fig. 2f). Even though granulocytes were not identified in the H\&E sections, some cells stained positive for anti-myeloperoxidase, a granulocyte marker, in the IF pictures (Fig. 2f). Statistical analysis of the prevalence of infiltrating cells showed statistically significant $(P \leq 0.05)$ elevated levels of T-lymphocytes, von Willebrand factor (vWf) positive cells, macrophages, and granulocytes compared to HAM, but no significant difference compared to other groups (Fig. 2g). The plasma cell staining was higher in the RHCI group compared to both HAM and fibrin glue. No significant differences among groups were found in the myofibroblast counts.

\section{In vivo Performance in Minipigs}

Results for the clinical observations of syngeneic grafts and their unoperated contralateral untreated corneas were previously reported in McTiernan et al. as the same four animals served as controls for the two different studies [21]. Clinical results are summarized in Fig. 3. At 6 weeks post-operation, the RHCI implants were completely covered with regenerated epithelium, as noted from clinical observation and slit lamp. The corneal thickness in the RHCI group was close to unoperated ranges at 3 months, and indistinguishable from unoperated by 6 months onwards (Fig. 3a). Even though there looks to be a trend towards a thicker cornea in the RHCI group compared to the syngeneic graft, this difference was statistically non-significant.

There is a statistically significant amount of haze in the RHCI group (Fig. 3c) that decreased with time and was scored at zero in two out of the four animals at the 12-month point. At the 12-month point, the difference between groups is not statistically significant (Tukey's multiple comparisons $\alpha=0.05$ ).

The subepithelial nerve plexus recovered fast, and at 12 months, nerve plexus morphology in the RHCI group resembled that of unoperated animals as seen in IVCM in Fig. 4. Nerve density of the corneal stroma, as noted based on IVCM, was close to normal ranges in both groups at 3 months onwards (Fig. 3e). One animal in the RHCI group had haze in the regenerated cornea that did not allow for imaging to obtain nerve counts. The missing datapoints were taken into account by using a mixed model rather than ANOVA for this comparison. All the pigs regained touch response in the operated eye prior to 12 months. Statistical analysis could not be performed due to uneven state of sedation of the animals during measurement.

Intraocular pressure was indistinguishable from unoperated eyes at all timepoints in both the RHCI group and the syngeneic grafts (Fig. 3b). The normal intraocular pressure (IOP) shows that there is no weakening of the cornea capable of causing long-term distortions or leakage, nor was the implant too rigid to cause increased pressure.

$H \& E$ sections showed that the stratified corneal epithelium and stroma have regenerated in the neo-corneas of all four pigs, to resemble those in the syngeneic grafts and untreated, healthy controls (Fig. 5g-1). Immunohistochemical staining showed full regeneration of a mucin-positive tear film and fully differentiated epithelial cells (Fig. 5). Antibodies against smooth muscle actin ( $\alpha$-SMA) showed the presence of positive cells in RHCI, most likely myofibroblasts, but not the syngeneic or control untreated corneas (Fig. 5y-ad).

\section{Discussion}

Plant-derived RHCI was successfully fabricated into aligned and random hydrogels. The RHCI was aligned to mimic the highly aligned, lamellar structure of collagen in the cornea. Unfortunately, the aRHCI hydrogels were opaque. Despite the same net weight of RHCI in the random and aligned gels, the aRHCI gels were thicker as the collagen assembled into thicker fibrils with variable spacing in between fibers. They also demonstrated extreme transverse mechanical weakness. The optical and mechanical deficits of the hydrogels in comparison to cornea is most likely due to the structural deficits of RHCI-only fibrils and a lack of perpendicular structural elements (for review, see Espana et al.) [24]. In the cornea, collagen protofibril formation is regulated through the incorporation of collagen $\mathrm{V}$ to create uniform protofibrils for subsequent fibrillar assembly [24]. In the absence of collagen V, collagen I produces thicker, branching, heterogeneous fibers like the ones observed by AFM and TEM in this study. These fibers are known to be incompatible with corneal transparency. Collagen fiber spacing also contributes to the aRHCI structural deficits. The spacing of corneal collagen fibrils is regulated by small leucine-rich proteoglycans, preventing the compaction of the collagen fibrils seen in the aRHCI [24]. The high aRHCI fibril density also contributes to the limitations 

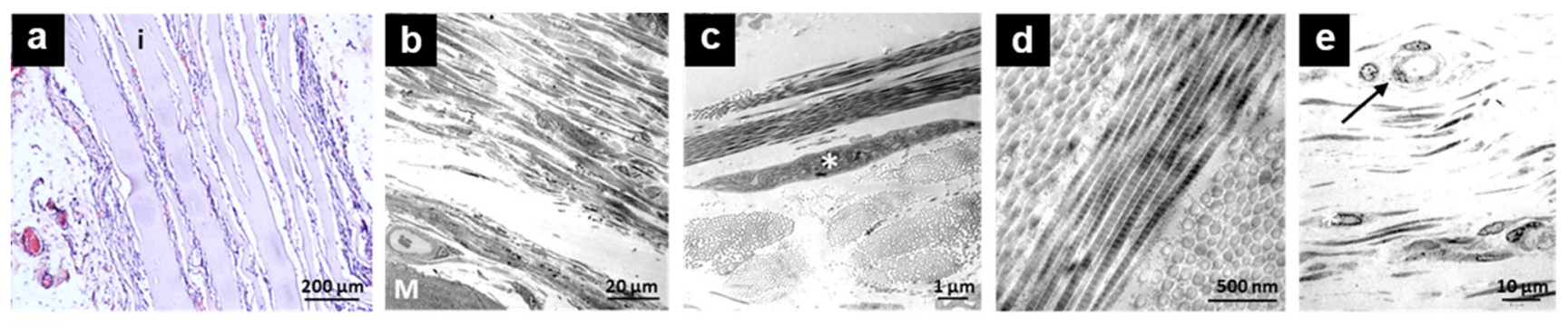

f
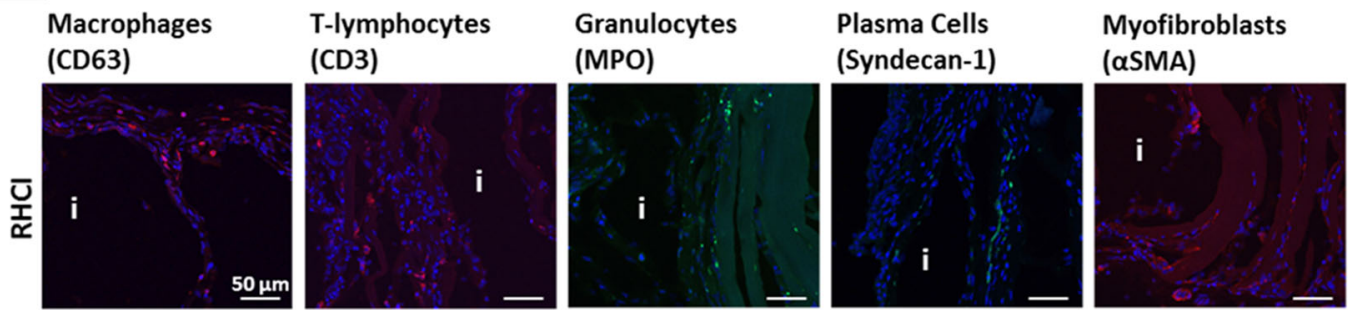

Blood vessels
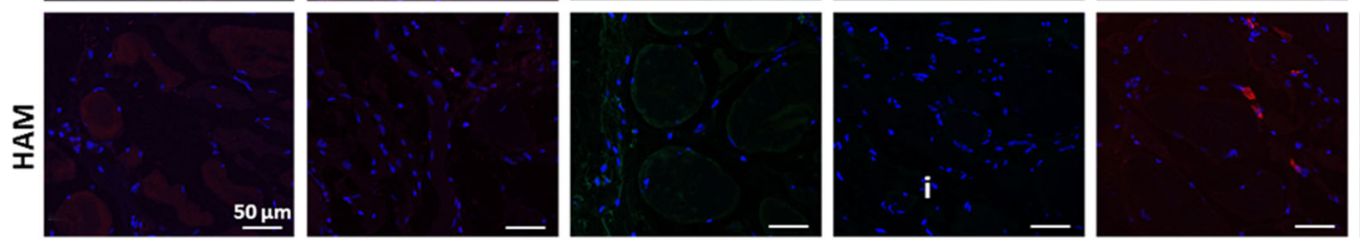

(vWF)

(9)
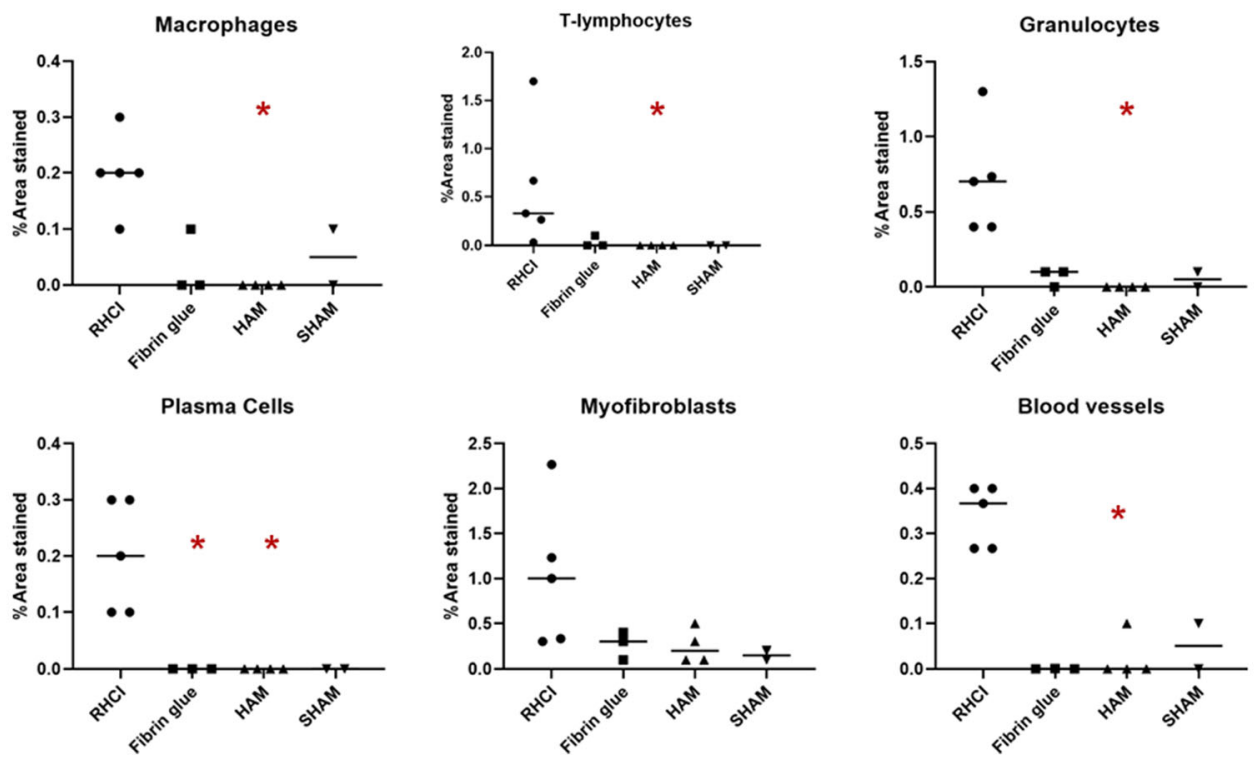

Fig. 2 Subcutaneous RHCI hydrogel implantation in rats for 3 months. a H\&E staining showing a delaminating RHCI implant (i) with infiltration of blood vessels, leukocytosis, and fibrosis. b Low magnification TEM image of recovered implants shows the implanted hydrogel in close approximation to the subcutaneous muscle (M). c Detailed TEM imaging shows clear fibroblast infiltration (*) and deposition of collagen fibers in bundles. d Typical collagen periodic D-banding is present in collagen

fibers. e Region of implant degradation characterized by loosely packed collagen fibers, interspersed with capillaries (arrow), leucocytes, and fibroblasts (*). f Representative images of immunostained samples for macrophages, T-cells, granulocytes, plasma cells, myofibroblasts, and blood vessels in implants. g Graphs showing the percentage of immune-positive staining areas for each marker, a red $*$ indicates a significant difference from the RHCI group $(p<0.05)$

in light transmission through the hydrogels. Finally, the collagen fibrils in each lamellar layer of the cornea are aligned in different directions, providing uniform mechanical strength throughout the entire cornea [24]. The single layer, shear force alignment method used here was insufficient to produce the structural complexity required to fully mimic the human cornea and will undergo future modifications, in order to improve the transparency of the material. 
A

Pachymetry

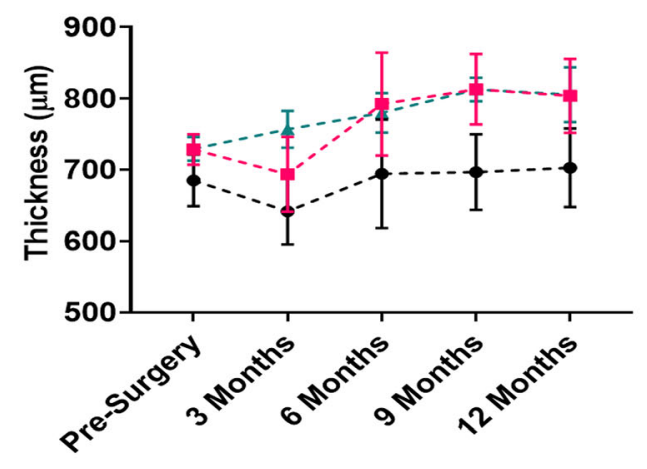

C

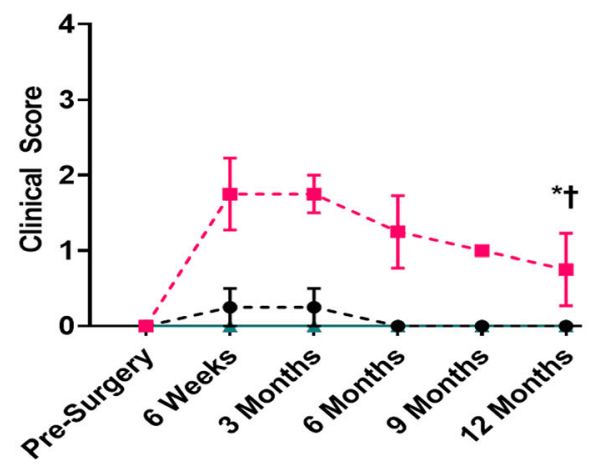

E

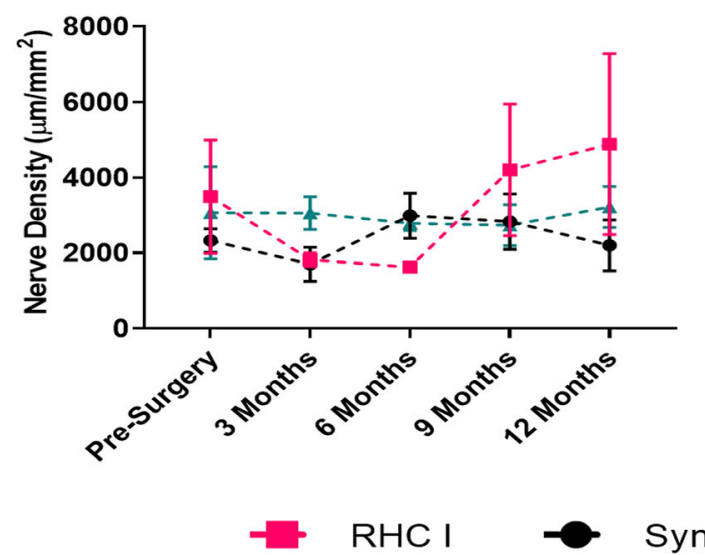

B

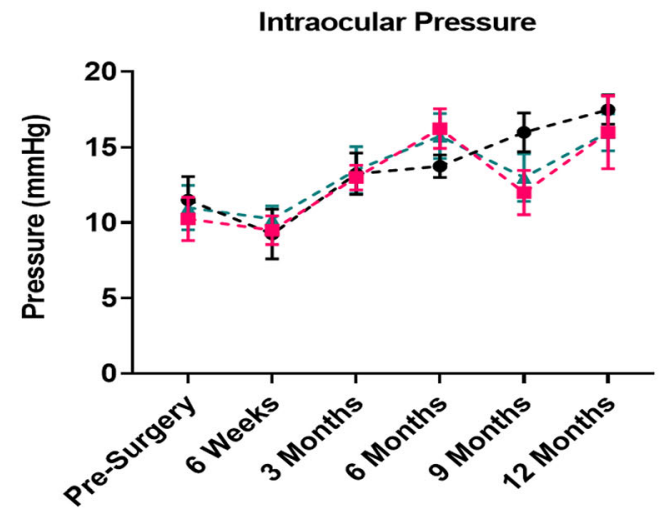

Implant Neovascularization

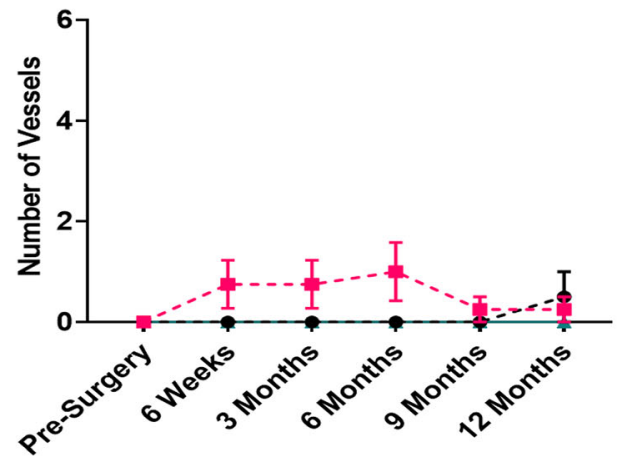

$\mathbf{F}$

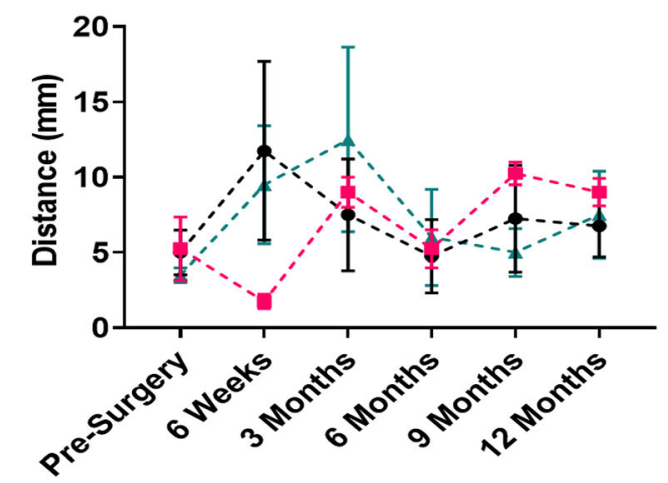

Fig. 3 Clinical results comparing RHCI grafted corneas with syngeneic grafts and untreated controls. Please note that the plots for the syngeneic grafts and unoperated controls were previously reported in McTiernan et al., as the same animals served as benchmarks for both studies. A Pachymetry results indicating corneal thickness was comparable to unoperated controls. B Central corneal haze was reported according to a modified variant of McDonald-Shadduck scale of $0-4$ (from least to most haze). Haze peaked between 6 weeks and 3 months and then decreases. By 12 months, the difference among groups have decreased to a nonsignificant level (by Tukey's multiple comparisons). C The excised corneal

By contrast, the rRHCI hydrogels were transparent and mechanically stronger in all directions. When compared to nerves in the RHCI group showed penetrance into the graft area after six months of tissue regeneration. Overall, the regenerated nerve density for RHCI and syngeneic grafts was equivalent to the unoperated eye at 12 months. D There was increased corneal neovascularization observed. This is also shown by an ANOVA, but the post hoc test could however not identify any particular time-point where there was a difference between the RHCI group and the groups (B) Intraocular pressure was similar in all animals, with an increase over time as the animals matured. C, F The Schirmer tear tests showed no marked changes in tear production in any group

previously tested collagen hydrogels, the transparency of random RHCI hydrogels was equivalent to that of porcine 
Fig. 4 In vivo confocal microscope images of RHCI and syngeneic grafted corneas, compared to unoperated healthy corneas. Scale bars, $100 \mu \mathrm{m}$. Insets, $100 \times 100 \mu \mathrm{m}$. The insets show epithelial cell morphology in detail. Sub-epithelial nerves (arrows) in regenerated RHCI implanted corneas form parallel bundles like those of unoperated corneas, while those of syngeneic grafts were disorganized. Stromal keratocytes were present in all three groups. The endothelial layers of operated corneas retained a healthy morphology

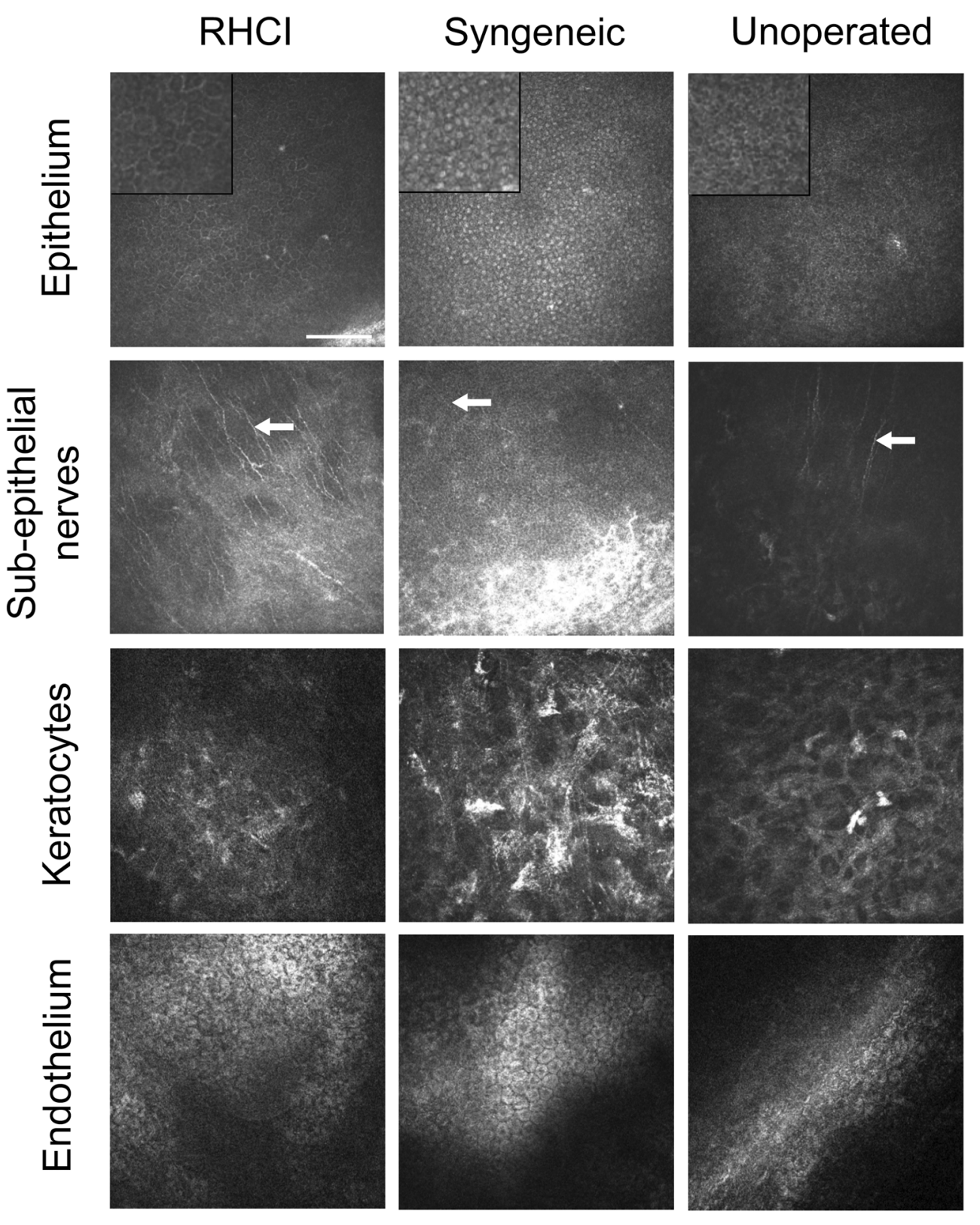

collagen type I, yeast-sourced RHCI, RHCIII, and collagen-mimicking hydrogels [25-29]. Moreover, rRHCI hydrogels outperformed HAM and rat tail collagen type I gels in terms of light transmission and refractive index [30]. In this study, RHCI hydrogels degraded faster in collagenase than HAM. The data on HAM degradation times should however be interpreted carefully; in this study, HAM degraded much slower than earlier reported degradation times of 6-13 h [18, 20]. The variability in degradation speeds of HAM underscores the inconsistent state of donated HAM and the need for well-standardized carrier materials in ocular tissue engineering. Aligned RHCI degraded too quickly for future clinical purposes without further modifications to its chemical formulation [31]. The difference in degradation time between both types of RHCI hydrogel may be attributed to the difference in collagen fibril density. One would assume, it would take longer to degrade the densely packed fibers in the aRHCI; however, without the interpenetrating fibrils holding the aligned fibers together, the hydrogel disintegrates into isolated fibers. Given the dense fibrillar network in the rRHCI hydrogels, these hydrogels degraded slower than aRHCI. Collagenase resistance of rRHCI was comparable to reported resistance of pure RHCIII, porcine collagen type I, and rat tail collagen type I hydrogels, with $8 \mathrm{~h}, 12-20 \mathrm{~h}$, and $8 \mathrm{~h}$ degradation time respectively $[19,20,25,32]$.

As demonstrated by Griffith et al., collagen hydrogels have a tendency of being more resistant to microbial contamination when compared to HAM or the human cornea [20, 33]. As we inoculated membranes with $P$. aeruginosa, a bacterium responsible for causing severe corneal ulcers, results confirmed 

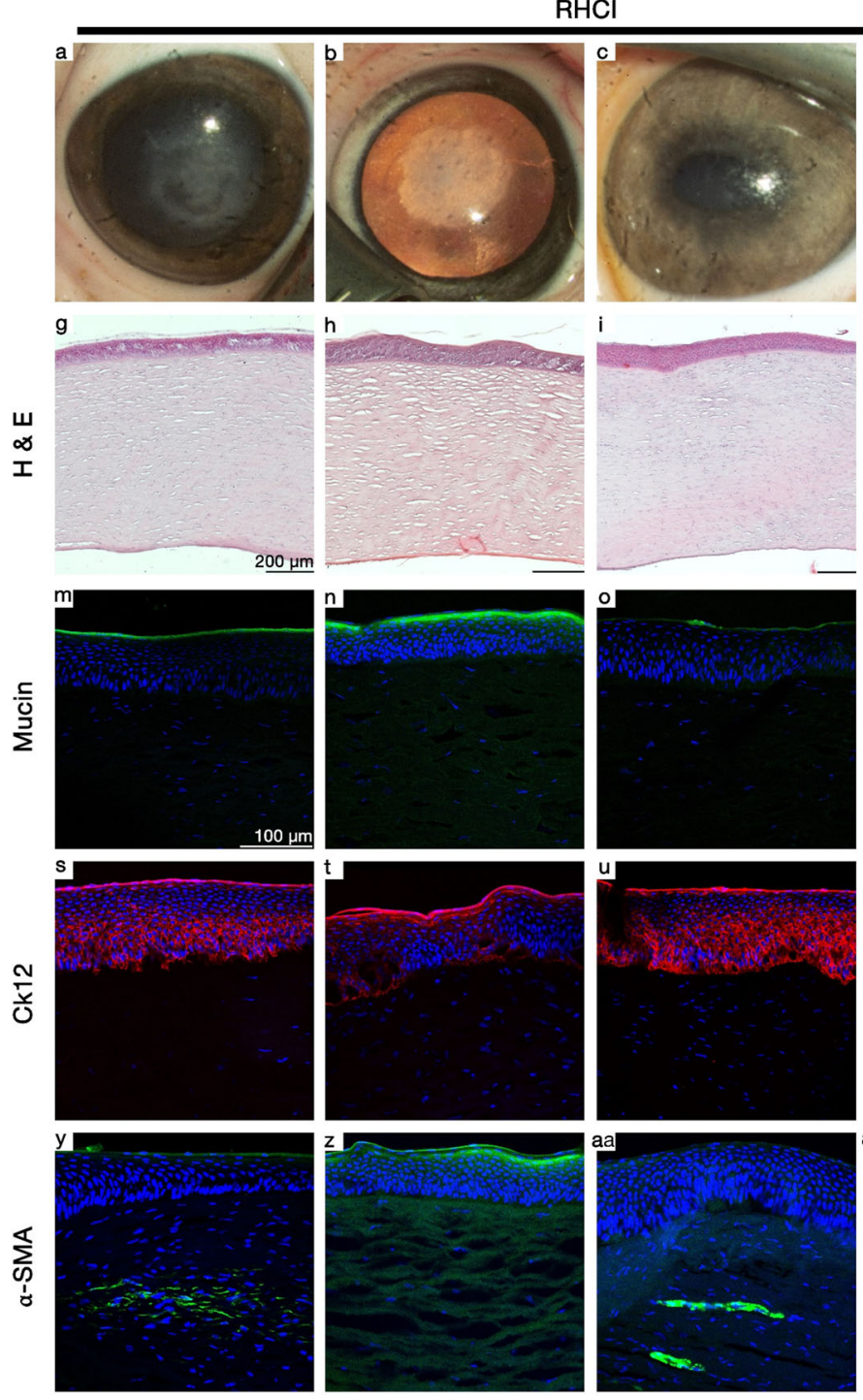

Fig. 5 Columns 1-4 shows each of the individual animals in the RHCI group. Column 5 shows representative images from the syngeneic graft. Column 6 shows representative images from unoperated controls. a-f Eyes of all RHCI grafted pigs and controls, showing haze in $\mathbf{a}$ and $\mathbf{b}$. g-l Hematoxylin and eosin stained sections show morphologies of regenerated neo-corneas are comparable to those of the controls. Mucin

the relative microbial resistance of RHCI compared to amniotic membrane. Microbial contamination of grafts is a serious and widespread problem in ocular surgery because it often causes devastating infections and loss of implanted material [20]. With a 100-fold resistance to microbial infection, RHCI has a clear advantage over HAM in a clinical setting, where substrates are manipulated repeatedly in clean rooms prior to transport to the operating theater.

By implanting RHCI hydrogels subcutaneously in rats, we demonstrated that they were biocompatible in mammals and elicit minimal inflammation. Furthermore, the recovered hydrogel implants had initiated partial degradation. As we and other groups previously reported intact hydrogel retrieval after

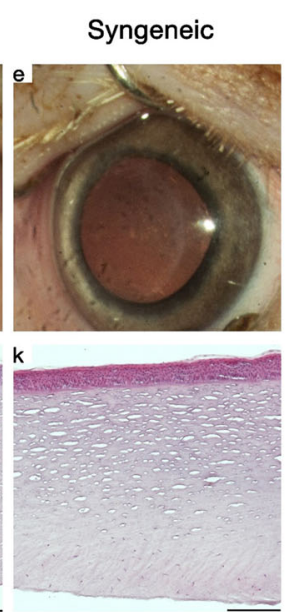

Unoperated
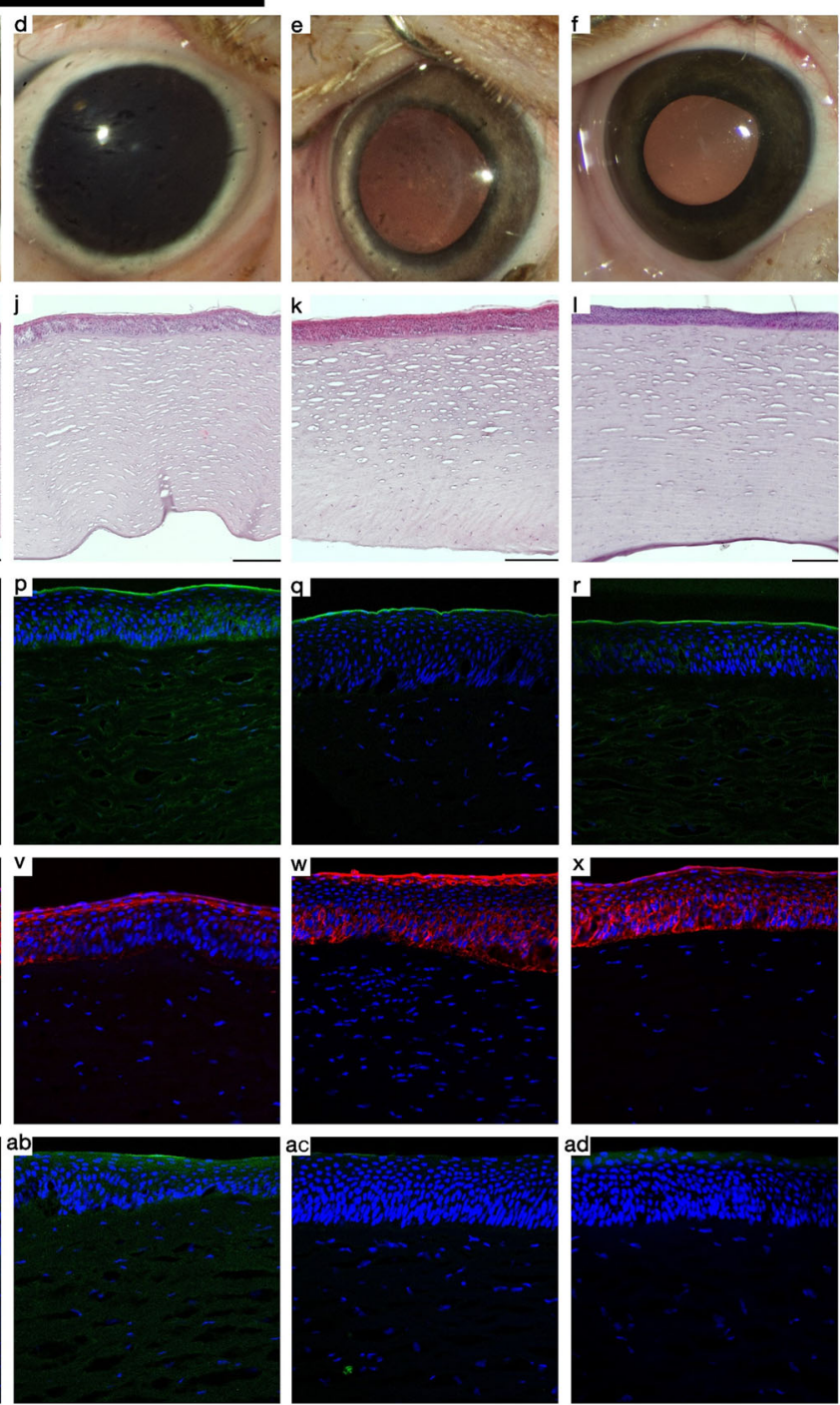

staining (m-r) shows that the animals in the RHCI group have intact tear films. Cytokeratin 12(Ck12) staining ( $\mathbf{s}-\mathbf{x})$ shows differentiated corneal epithelial cells in all samples. $\mathbf{y}$-ad $\alpha$-Smooth muscle actin ( $\alpha$-SMA), a myofibroblast marker shows the presence of activated fibroblasts in RHCI corneas, particularly in the ones with haze

90 days of implantation, this might indicate that additional crosslinking or incorporation of interpenetrating phospholipids to RHCI might improve the stability of the implant $[19,25,27,28,34]$. There was formation of new collagen fibers within the implants. This suggests that infiltrating fibroblasts had begun to remodel the implant. Similar observations have been made in corneal implants that were implanted in pigs, where host keratocytes remodeled the implants' fibrillar structure [19, 35]. By computer analysis of immunofluorescent images, we were able to quantify leukocytosis and neovascularization of retrieved implants and compare results to the isoproterenol-based H\&E grading system. Interestingly, no granulocytes could be identified in H\&E sections, whereas 
anti-myeloperoxidase, a marker for granulocytes, showed to be focally positive by immunohistochemistry, showing that H\&E histopathological examination should be supplemented by immunohistochemistry using known markers for target cells. In 2016, Van Essen et al. published their results on automated analysis of IF area staining for fish scale-derived collagen matrices that were implanted subcutaneously in rats for 11 weeks [36]. With reported area of stain of $<1 \%$ for macrophages and T-lymphocytes, results are very similar to the values we found. Moreover, area of stain for granulocytes appears to be lower for RHCI hydrogels compared to reported values for fish scale matrices. The presence of $\alpha$-SMA positive cells that are myofibroblast-like in the RHCI corneas are in keeping with the haze that was noted. They also correspond to the results from the subcutaneous study showing that RHCI was a mild irritant. It is possible that there may be traces of plant material that contributed to the irritation and activation of stromal cells.

Although there are collagens that are derived from bovine and porcine skin which are widely available and offer a potentially unlimited supply source, an important consideration for the use of xeno-derived collagen is the potential risk for allergic reactions to xenogeneic biomaterials [37] and zoonotic pathogen transmission [38] if tight processing controls are not adhered to. Overall, plant-derived RHCI was largely biocompatible and immune compatible. RHCI hydrogels promoted regeneration of corneal tissue and nerves when grafted into the corneas of minipigs. Finally, application of RHCI should not solely be restricted to ocular regeneration, as collagen type I is abundantly present throughout the body. Aligned RHCI, with its unique collagen alignment, might find its way in musculoskeletal tissue engineering, whereas random hydrogels might be explored in cutaneous or cardiac regeneration [39-41].

\section{Conclusions}

RHCI hydrogels made from random collagen fibrils were biocompatible and promoted corneal regeneration in minipigs. This shows that the plant-derived collagen serves as a viable source of collagen for tissue engineering and regenerative medicine applications, such as for promoting in situ tissue regeneration of the cornea after further optimization and testing.

Supplementary Information The online version contains supplementary material available at https://doi.org/10.1007/s40883-021-00220-3.

Acknowledgements We thank Dr. Christopher McTiernan, University of Ottawa Heart Institute, for the nerve counts in Fig. 4E. We acknowledge Science Advances (AAAS) for reproduction of results in Fig. 4 from McTiernan et al. We thank Prof. Dr. Sorcha Ní Dhubhghaill for her help with the pig surgeries, Prof. Dr. Nadia Zakaria for administration and logistics, and Ms. Nezahat Bostan and Ms. Eline Melsbach for procurement of the amniotic membranes and cadaveric donor eyes. Ms. Sofie Thys is acknowledged for performing the scanning electron microscopy and Ms. Bieke Vanhoutte for assisting in the microbial challenge testing. The tobacco plant in the graphical abstract is adapted from "Tobacco and its adulterations", this rendering is in the public domain [42].

Funding Open access funding provided by Linkoping University. This research was funded with research grants from The Research Foundation Flanders (\#FWO - 11ZB315N) to MH; EuroNanoMed II (G0D5615N to MJT) for the REGENERATE project in partnership with the Swedish Research Council (529-2014-7490 to MKL) and the Research Council of Lithuania (EuroNanoMed2-01/2015 to RV), and the European Cooperation in Science and Technology (EU-COST BM1302). MG acknowledges salary and research support from her Canada Research Chair Tier 1 in Biomaterials and Stem Cell in Ophthalmology and a Caroline Durand Foundation Research Chair in Cellular Therapy in the Eye, as well as a Honorary Professorship at the School of Optometry and Vision Science, Cardiff University. FS is supported by an NSERC Canada doctoral studentship.

Data Availability Data collected and analyzed for this study are provided in the Electronic Supplemental Material. Other data generated during the current study are available from the corresponding authors on reasonable request. RHCI available from CollPlant Ltd.

\section{Declarations}

Ethics Approval The Declaration of Helsinki guidelines were applied and were approved by the Antwerp University Hospital Ethical Committee (EC: 14/30/319) for use of human amniotic membrane. Animal experiments in rats and Göttingen minipigs were performed in accordance with the Association for Research in Vision and Ophthalmology Statement for the Use of Animals in Ophthalmic and Vision Research and the Swedish Animal Welfare Ordinance and the Animal Welfare Act, after ethical approval was acquired from the local Linkoping ethical committee (Linköpings Djurförsöksetiska Nämnd).

Competing Interests Dr. Oded Shoseyov is a founder and chief scientist at CollPlant which produces and markets tobacco-derived RHCI. Other authors have no competing interests.

Open Access This article is licensed under a Creative Commons Attribution 4.0 International License, which permits use, sharing, adaptation, distribution and reproduction in any medium or format, as long as you give appropriate credit to the original author(s) and the source, provide a link to the Creative Commons licence, and indicate if changes were made. The images or other third party material in this article are included in the article's Creative Commons licence, unless indicated otherwise in a credit line to the material. If material is not included in the article's Creative Commons licence and your intended use is not permitted by statutory regulation or exceeds the permitted use, you will need to obtain permission directly from the copyright holder. To view a copy of this licence, visit http://creativecommons.org/licenses/by/4.0/.

\section{References}

1. Gain P, Jullienne R, He Z, Aldossary M, Acquart S, Cognasse F, et al. Global survey of corneal transplantation and eye banking. JAMA Ophthalmol. 2016;134(2):167-73. https://doi.org/10.1001/ jamaophthalmol.2015.4776. 
2. Holak SA, Holak HM, Bleckmann H. AlphaCor keratoprosthesis: postoperative development of six patients. Graefe's Arch Clin Exp Ophthalmol $=$ Albrecht von Graefes Archiv für klinische und experimentelle Ophthalmologie. 2009;247(4):535-9. https://doi. org/10.1007/s00417-008-0964-7.

3. Zerbe BL, Belin MW, Ciolino JB, Boston Type 1 Keratoprosthesis Study G. Results from the multicenter Boston Type 1 Keratoprosthesis Study. Ophthalmology. 2006;113(10):1779.e1-7. https://doi.org/10.1016/j.ophtha.2006.05.015.

4. Casey TA. Osteo-odonto-keratoprosthesis. Proc R Soc Med. 1966;59(6):530-1.

5. Schrage N, Hille K, Cursiefen C. Aktuelle Versorgungsmöglichkeiten mit Keratoprothesen. Ophthalmologe. 2014;111(11):1010-8. https://doi.org/10. 1007/s00347-013-3009-5.

6. Ghezzi CE, Rnjak-Kovacina J, Kaplan DL. Corneal tissue engineering: recent advances and future perspectives. Tissue Eng B Rev. 2015;21(3):278-87. https://doi.org/10.1089/ten.TEB.2014. 0397.

7. Griffith M, Alarcon EI, Brunette I. Regenerative approaches for the cornea. J Intern Med. 2016;280(3):276-86. https://doi.org/10.1111/ joim. 12502 .

8. Fagerholm P, Lagali NS, Merrett K, Jackson WB, Munger R, Liu $\mathrm{Y}$, et al. A biosynthetic alternative to human donor tissue for inducing corneal regeneration: 24-month follow-up of a phase 1 clinical study. Sci Transl Med. 2010;2(46):46ra61. https://doi.org/10.1126/ scitranslmed.3001022.

9. Yang C, Hillas P, Tang J, Balan J, Notbohm H, Polarek J. Development of a recombinant human collagen-type III based hemostat. J Biomed Mater Res Part B Appl Biomater. 2004;69(1):1824. https://doi.org/10.1002/jbm.b.20030.

10. Vuorela A, Myllyharju J, Nissi R, Pihlajaniemi T, Kivirikko KI. Assembly of human prolyl 4-hydroxylase and type III collagen in the yeast Pichia pastoris: formation of a stable enzyme tetramer requires coexpression with collagen and assembly of a stable collagen requires coexpression with prolyl 4-hydroxylase. EMBO J. 1997;16(22):6702-12. https://doi.org/10.1093/emboj/16.22.6702.

11. Stein H, Wilensky M, Tsafrir Y, Rosenthal M, Amir R, Avraham T, et al. Production of bioactive, post-translationally modified, heterotrimeric, human recombinant type-I collagen in transgenic tobacco. Biomacromolecules. 2009;10(9):2640-5. https://doi.org/ 10.1021/bm900571b.

12. Abir R, Stav D, Taieb Y, Gabbay-Benziv R, Kirshner M, BenHaroush A, et al. Novel extra cellular-like matrices to improve human ovarian grafting. J Assist Reprod Genet. 2020;37(9):210517. https://doi.org/10.1007/s10815-020-01832-4.

13. Andrée B, Ichanti H, Kalies S, Heisterkamp A, Strauß S, Vogt P-M, et al. Formation of three-dimensional tubular endothelial cell networks under defined serum-free cell culture conditions in human collagen hydrogels. Sci Rep. 2019;9(1):5437. https://doi.org/10. 1038/s41598-019-41985-6.

14. Haagdorens M, Cèpla V, Melsbach E, Koivusalo L, Skottman H, Griffith M, et al. In vitro cultivation of limbal epithelial stem cells on surface-modified crosslinked collagen scaffolds. Stem Cells Int. 2019;2019:7867613-7. https://doi.org/10.1155/2019/7867613.

15. Zhong SP, Teo WE, Zhu X, Beuerman R, Ramakrishna S, Yung LYL. Development of a novel collagen-GAG nanofibrous scaffold via electrospinning. Mater Sci Eng C. 2007;27(2):262-6. https:// doi.org/10.1016/j.msec.2006.05.010

16. Torbet J, Malbouyres M, Builles N, Justin V, Roulet M, Damour O, et al. Orthogonal scaffold of magnetically aligned collagen lamellae for corneal stroma reconstruction. Biomaterials. 2007;28(29): 4268-76. https://doi.org/10.1016/j.biomaterials.2007.05.024.

17. Zakaria N, Koppen C, Van Tendeloo V, Berneman Z, Hopkinson A, Tassignon MJ. Standardized limbal epithelial stem cell graft generation and transplantation. Tissue Eng Part C, Methods. 2010;16(5):921-7. https://doi.org/10.1089/ten.TEC.2009.0634.

18. Van den Bogerd B, Ní Dhubhghaill S, Zakaria N. Characterizing human decellularized crystalline lens capsules as a scaffold for corneal endothelial tissue engineering. J Tissue Eng Regen Med. 2018;12(4):2020-8. https://doi.org/10.1002/term.2633.

19. Koulikovska M, Rafat M, Petrovski G, Veréb Z, Akhtar S, Fagerholm P, et al. Enhanced regeneration of corneal tissue via a bioengineered collagen construct implanted by a nondisruptive surgical technique. Tissue Eng A. 2015;21(5-6):1116-30. https://doi. org/10.1089/ten.tea.2014.0562.

20. Dravida S, Gaddipati S, Griffith M, Merrett K, Lakshmi Madhira S, Sangwan VS, et al. A biomimetic scaffold for culturing limbal stem cells: a promising alternative for clinical transplantation. J Tissue Eng Regen Med. 2008;2(5):263-71. https://doi.org/10.1002/term. 91.

21. McTiernan CD, Simpson FC, Haagdorens M, Samarawickrama C, Hunter D, Buznyk O, et al. LiQD Cornea: Pro-regeneration collagen mimetics as patches and alternatives to corneal transplantation. Sci Adv. 2020;6(25):eaba2187. https://doi.org/10.1126/sciadv. aba2187.

22. Jangamreddy JR, Haagdorens MKC, Islam MM, Lewis P, Samanta A, Fagerholm P, et al. Short peptide analogs as alternatives to collagen in pro-regenerative corneal implants. Acta Biomater. 2018;69:120-30. https://doi.org/10.1016/j.actbio.2018.01.011.

23. Meijering E, Jacob M, Sarria JC, Steiner P, Hirling H, Unser M. Design and validation of a tool for neurite tracing and analysis in fluorescence microscopy images. Cytometry A. 2004;58(2):16776. https://doi.org/10.1002/cyto.a.20022.

24. Espana EM, Birk DE. Composition, structure and function of the corneal stroma. Exp Eye Res. 2020;198:108137. https://doi.org/10. 1016/j.exer.2020.108137.

25. Merrett K, Liu W, Mitra D, Camm KD, McLaughlin CR, Liu Y, et al. Synthetic neoglycopolymer-recombinant human collagen hybrids as biomimetic crosslinking agents in corneal tissue engineering. Biomaterials. 2009;30(29):5403-8. https://doi.org/10.1016/j. biomaterials.2009.06.016.

26. Islam MM, Cèpla V, He C, Edin J, Rakickas T, Kobuch K, et al. Functional fabrication of recombinant human collagenphosphorylcholine hydrogels for regenerative medicine applications. Acta Biomater. 2015;12:70-80. https://doi.org/10.1016/j. actbio.2014.10.035.

27. Liu W, Deng C, McLaughlin CR, Fagerholm P, Lagali NS, Heyne $\mathrm{B}$, et al. Collagen-phosphorylcholine interpenetrating network hydrogels as corneal substitutes. Biomaterials. 2009;30(8):15519. https://doi.org/10.1016/j.biomaterials.2008.11.022.

28. Islam MM, Ravichandran R, Olsen D, Ljunggren M, Fagerholm P, Lee CJ, et al. Self-assembled collagen-like-peptide implants as alternatives to human donor corneal transplantation. RSC Adv. 2016;6(61):55745-9. https://doi.org/10.1039/C6RA08895C.

29. Rafat M, Xeroudaki M, Koulikovska M, Sherrell P, Groth F, Fagerholm P, et al. Composite core-and-skirt collagen hydrogels with differential degradation for corneal therapeutic applications. Biomaterials. 2016;83:142-55. https://doi.org/10.1016/j. biomaterials.2016.01.004.

30. Tidu A, Ghoubay-Benallaoua D, Lynch B, Haye B, Illoul C, Allain $\mathrm{JM}$, et al. Development of human corneal epithelium on organized fibrillated transparent collagen matrices synthesized at high concentration. Acta Biomater. 2015;22:50-8. https://doi.org/10.1016/j. actbio.2015.04.018

31. Spoerl E, Wollensak G, Reber F, Pillunat L. Cross-linking of human amniotic membrane by glutaraldehyde. Ophthalmic Res. 2004;36(2):71-7. https://doi.org/10.1159/000076884.

32. Koh LB, Islam MM, Mitra D, Noel CW, Merrett K, Odorcic S, et al. Epoxy cross-linked collagen and collagen-laminin Peptide 
hydrogels as corneal substitutes. J Funct Biomater. 2013;4(3):16277. https://doi.org/10.3390/jfb4030162.

33. Deng C, Li F, Hackett JM, Chaudhry SH, Toll FN, Toye B, et al. Collagen and glycopolymer based hydrogel for potential corneal application. Acta Biomater. 2010;6(1):187-94. https://doi.org/10. 1016/j.actbio.2009.07.027.

34. Merrett K, Fagerholm P, McLaughlin CR, Dravida S, Lagali N, Shinozaki N, et al. Tissue-engineered recombinant human collagen-based corneal substitutes for implantation: performance of type I versus type III collagen. Invest Ophthalmol Vis Sci. 2008;49(9):3887-94. https://doi.org/10.1167/iovs.07-1348.

35. Liu W, Merrett K, Griffith M, Fagerholm P, Dravida S, Heyne B, et al. Recombinant human collagen for tissue engineered corneal substitutes. Biomaterials. 2008;29(9):1147-58. https://doi.org/10. 1016/j.biomaterials.2007.11.011.

36. van Essen TH, van Zijl L, Possemiers T, Mulder AA, Zwart SJ, Chou $\mathrm{CH}$, et al. Biocompatibility of a fish scale-derived artificial cornea: cytotoxicity, cellular adhesion and phenotype, and in vivo immunogenicity. Biomaterials. 2016;81:36-45. https://doi.org/10. 1016/j.biomaterials.2015.11.015.

37. Rieder E, Steinacher-Nigisch A, Weigel G. Human immune-cell response towards diverse xenogeneic and allogeneic decellularized biomaterials. Int J Surg. 2016;36(Pt A):347e51. https://doi.org/10. 1016/j.ijsu.2016.06.042.

38. Silvipriya K, Kumar K, Bhat A, Kumar B, John A, Lakshmanan P. Collagen: animal sources and biomedical application. J Appl Pharm Sci. 2015;5:123-7. https://doi.org/10.7324/JAPS.2015.50322.

39. Docheva D, Müller SA, Majewski M, Evans CH. Biologics for tendon repair. Adv Drug Deliv Rev. 2015;84:222-39. https://doi. org/10.1016/J.ADDR.2014.11.015.

40. Shahrokhi S, Arno A, Jeschke MG. The use of dermal substitutes in burn surgery: acute phase. Wound repair and regeneration : official publication of the Wound Healing Society [and] the European Tissue Repair. Society. 2014;22(1):14-22. https://doi.org/10.1111/ wrr.12119.

41. Kochegarov A, Lemanski LF. New trends in heart regeneration: a review. J Stem Cells Regen Med. 2016;12(2):61-8. https://doi.org/ 10.46582/jsrm. 1202010.

42. Prescott HP. Tobacco, and its adulterations. Lancet. 1853;62(1561): 103-7. https://doi.org/10.1016/S0140-6736(02)39999-9.

Publisher's Note Springer Nature remains neutral with regard to jurisdictional claims in published maps and institutional affiliations. 